\title{
HIPERTENSÃO PORTAL ESQUISTOSSOMÓTICA: influência do fluxo sangǘneo portal nos níveis séricos das enzimas hepáticas
}

\author{
Antonio ALVES Jr. ${ }^{\mathbf{1}}$, Dercílio Alves FONTES ${ }^{\mathbf{1}}$, \\ Valdinaldo Aragão de MELO ${ }^{1}$, Marcel Cerqueira C. MACHADO ${ }^{2}$, \\ Josilda Ferreira CRUZ ${ }^{1}$ e Egmond Alves Silva SANTOS $^{1}$
}

RESUMO - Objetivo - Avaliar a relação entre o fluxo sangüíneo portal e o perfil laboratorial hepático em pacientes com hipertensão portal esquistossomótica. Pacientes e Métodos - Estudaram-se 64 pacientes com hipertensão portal esquistossomótica, sendo 19 não-operados, 23 submetidos a desconexão ázigo-portal com esplenectomia e 22 submetidos a derivação esplenorrenal distal. Avaliou-se o perfil laboratorial hepático através da dosagem sérica de albumina, transaminases glutâmico-oxalacética e glutâmico-pirúvica, bilirrubinas direta e indireta, fosfatase alcalina, gama-glutamil transferase e avaliação do tempo de protrombina. O fluxo portal foi avaliado por Doppler. Os resultados foram analisados através de regressão linear, coeficiente de correlação de Pearson, teste do Qui-quadrado e análise de variância de um via com pós-teste de Tukey. Resultados - Evidenciou-se que somente a gama-glutamil transferase teve correlação significativa com o fluxo portal. No cotejo dos quartis, também, somente a gama-glutamil transferase mostrou resultado significativo, em que se constatou que o quarto quartil, de maior fluxo portal e formado em sua maioria por pacientes não operados, também foi o de maior valor médio de gama-glutamil transferase e significativamente maior que o primeiro e terceiro quartis. Conclus ões Estes dados sugerem que: quanto maior o fluxo sangüíneo portal, maior o nível sérico de gama-glutamil transferase; a gama-glutamil transferase é a variável da avaliação do perfil hepático mais representativa da influência do fluxo portal na atividade funcional hepática nos pacientes com esquistossomose hepatoesplênica, e é possível que as cirurgias, através de suas modificações hemodinâmicas (diminuição da congestão), sejam também benéficas por diminuírem o grau de colestase presente ou em regredirem a indução microssomal.

DESCRITORES - Hipertensão portal. Veia porta. Testes de função hepática.

\section{INTRODUÇÃO}

A Organização Mundial de Saúde estima que a esquistossomose acometa mais de 200 milhões de pessoas em todo o mundo, representando a principal causa mundial de hipertensão portal e acometendo geralmente indivíduos jovens ${ }^{(24,30)}$. No Brasil, é estimado o número de 8 a 18 milhões de infestados pelo Schistosoma mansoni ${ }^{(30)}$, atingindo principalmente uma faixa de contínua endemicidade que se estende pelos Estados do Rio Grande do Norte, Paraíba, Pernambuco, Alagoas, Sergipe, Bahia e Minas Gerais ${ }^{(10)}$.

A hemorragia digestiva alta por varizes esofagogástricas é a principal manifestação da hipertensão portal esquistossomótica (HPE) e, também, a principal complicação com altos índices de morbimortalidade ${ }^{(12,29)}$.
Para evitar o ressangramento, existem na atualidade duas técnicas cirúrgicas mais praticadas e estudadas: a desconexão azigoportal com esplenectomia (DAPE) e a derivação esplenorrenal distal (DERD). O tratamento cirúrgico ideal ainda não foi atingido, estando as opções cirúrgicas entre o eficiente controle da recidiva hemorrágica, contudo com proibitivos índices de encefalopatia hepática (no caso da DERD), e o não aparecimento de encefalopatia, mas com taxas maiores de ressangramento digestivo (DAPE) ${ }^{(1,5,14,30,31)}$.

A avaliação da hemodinâmica hepática pode esclarecer sobre a fisiopatogenia e sobre as alterações observadas nos diferentes tipos de cirurgias $^{(9,31)}$. Os métodos mais usados para esta avaliação utilizam-se de medidas invasivas. Entretanto, através do surgimento da ultra-sonografia Doppler duplex, houve a possibilidade de obtenção de medidas não-invasivas da velocidade

Trabalho realizado pelo 'Departamento de Medicina da Universidade Federal de Sergipe, Aracaju, SE, e pela ${ }^{2}$ Disciplina de Transplante e Cirurgia de Fígado da Faculdade de Medicina da Universidade de São Paulo, São Paulo, SP.

Endereço para correspondência: Dr. Antonio Alves Júnior - Rua Tenente Wendel Quaranta, 1371 - Ed. Van Gogh, apt. 1001 - $49052-260$ - Aracaju, SE.

E-mail: aalves@infonet.com.br 
sangüínea, permitindo o cálculo do fluxo sangüíneo. Desta forma, obteve-se um exame de simples execução, baixo custo e não-invasivo, apresentando-se bastante útil na avaliação, planejamento terapêutico e acompanhamento do paciente com hipertensão portal ${ }^{(8,15,17,19,26,32,38)}$.

Muitos trabalhos têm estudado, em pacientes com hipertensão portal cirrótica, a dependência da função hepática ao fluxo sangüíneo hepático e ao fluxo sangüíneo portal (FP) ${ }^{(11,18,20,21,28)}$. Esses trabalhos evidenciam uma interferência importante do FP na atividade funcional do fígado. É conhecido, entretanto, que a hipertensão portal cirrótica é localizada nos sinusóides (hipertensão portal sinusoidal) e que se associa à lesão hepatocelular importante. Deste modo, a função destas células está comprometida e estas se tornam lábeis às mudanças de sua perfusão. Já os pacientes com HPE têm, geralmente, a arquitetura lobular e a função hepática mantidas, sendo as alterações vasculares hepáticas e do sistema porta os aspectos fundamentais mais expressivos na fisiopatologia da $\operatorname{HPE}^{(9,23)}$. Estes aspectos transformam a HPE num bom modelo para estudo da fisiologia do FP na atividade funcional do fígado.

Neste sentido e observando que existem poucos estudos na literatura abordando a relação da função hepática com o FP na $\mathrm{HPE}^{(7)}$, elaborou-se a presente pesquisa que teve como objetivos: 1) determinar se há correlação significativa entre o perfil hepático (avaliado através das dosagens de albumina (ALB), TGO, TGP, bilirrubina direta (BD) e indireta (BI), FA, GGT e tempo de protrombina (TP) e o FP, e 2) identificar qual a variável do perfil hepático mais representativa da influência do FP na atividade funcional hepática nos pacientes com HPE.

\section{PACIENTES E MÉTODOS}

Este estudo foi aprovado pelo Comitê de Ética do hospital e um consentimento escrito foi obtido de cada paciente. Realizou-se estudo retrospectivo com 64 pacientes com HPE, sendo 19 (29,69\%) nãooperados, 23 (35,94\%) submetidos a DAPE e 22 (34,37\%) submetidos a DERD. Os dados foram coletados no período entre maio de $1996 \mathrm{e}$ maio de 1999. Os pacientes foram agrupados e avaliados em quartis de acordo com o FP, conforme ilustrado na Figura 1.

A composição da casuística dos diferentes quartis foi homogênea, não havendo diferença significativa referente às médias de idade, peso e altura dos pacientes $(P>0,05)$ nos diferentes quartis, bem como referente ao tempo de pós-operatório $(P=0,1145)$.

Para compor os grupos, foram utilizados os seguintes critérios de inclusão: 1) idade entre 15 e 70 anos, 2) diagnóstico de esquistossomose hepatoesplênica com hipertensão portal, confirmado pelo achado endoscópico de varizes esofagogástricas, 3) antecedente de hemorragia digestiva alta, 4) intervalo mínimo de 15 dias entre o episódio hemorrágico e os exames de FP e do perfil hepático.

Consideraram-se critérios de exclusão: 1) antecedente de alcoolismo, 2) presença de cardiopatia ou pneumopatia clinicamente importantes, 3) evidência de qualquer outra hepatopatia, 4) presença

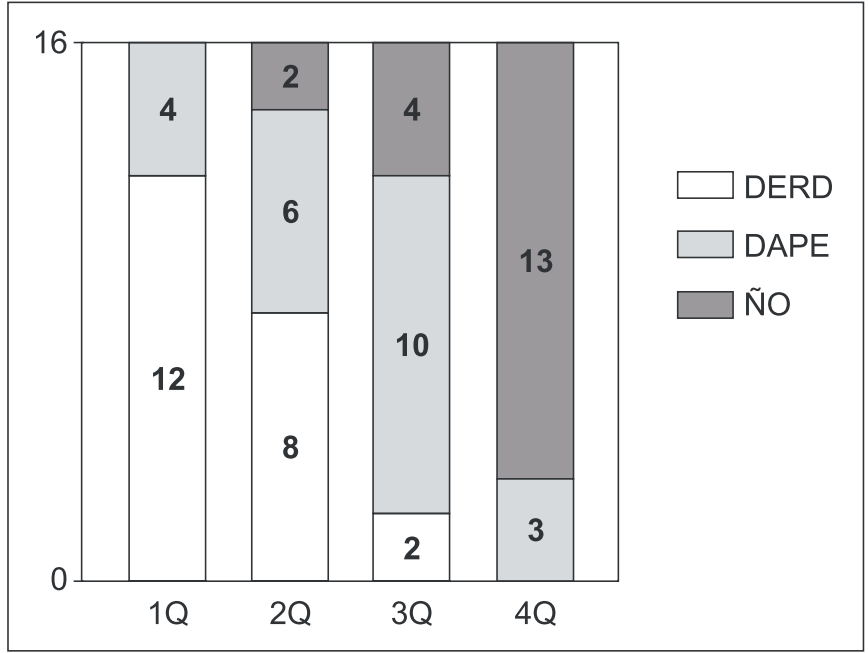

FIGURA 1 - Representação gráfica da composição dos quartis. 1Q: $\mathrm{FP}<672,79 \mathrm{~mL} / \mathrm{min} ; 2 \mathrm{Q}:$ FP entre 672,79 e $1.093,52$ $\mathrm{mL} / \mathrm{min}$; 3Q: FP entre 1.093,52 e 1.523,25 mL/min; 4Q: FP >1.523,25 $\mathrm{mL} / \mathrm{min}$. DERD = derivação esplenorrenal distal; DAPE = desconexão azigoportal com esplenectomia; $\tilde{N} O$ - não operados

de febre ou processo infeccioso agudo, 5) presença de trombose de veias esplênica ou mesentérica superior, 6) presença de trombose de anastomose esplenorrenal.

O perfil laboratorial hepático dos pacientes foi avaliado através dos valores obtidos com a dosagem sérica da ALB, transaminase glutâmico-oxalacética (TGO), transaminase glutâmico-pirúvica (TGP), BD e BI, fosfatase alcalina (FA) e gama-glutamil transferase (GGT). Essas dosagens foram realizadas pelo método colorimétrico automatizado do Cobas Mira Plus Chemistry System ${ }^{\circledR}$ (Roche Diagnostic System Incorporated). O TP ${ }^{(36)}$ também foi utilizado, sendo realizado o teste de Quick ${ }^{(25)}$.

O FP foi mensurado através do Doppler duplex colorido (Toshiba SSH 140 com transdutor eletrônico convexo de 3,75 MHz, indicado para o estudo de vasos em profundidade no organismo) realizado sempre pelo mesmo profissional e o mesmo aparelho para todos os pacientes, evitando o risco de variação entre observadores ${ }^{(33)}$. As medições foram feitas em triplicata (considerou-se a média das três aferições) e obtidas no tronco da veia porta antes da bifurcação, através de corte oblíquo subcostal direito ${ }^{(26,37)}$. Considerando a influência da fase digestiva sobre o fluxo venoso esplâncnico, cada paciente foi orientado a comparecer em jejum de 12 horas e a tomar 40 gotas de Luftal ${ }^{\mathbb{B}}$ (Bristol-Myers Squibb Brasil S/A, São Paulo, SP) na noite anterior e na manhã do exame. A posição padronizada para o estudo foi o decúbito dorsal horizontal assumido após 15 a 30 minutos de descanso (sentado sem atividade), para obviar a interferência postural e da atividade física ${ }^{(33)}$.

Os planos de corte ultra-sonográficos foram sempre os mesmos para todos os pacientes e seguiram a padronização da Organização 
Mundial da Saúde no uso da ultra-sonografia para avaliação do fígado, baço e sistema vascular esplâncnico na $\mathrm{HPE}^{(38)}$. Acrescente-se o fato de que os cortes aludidos foram sempre obtidos durante a mesma fase da respiração, evitando as variações com o ciclo respiratório.

Cada variável do perfil laboratorial hepático foi correlacionada com o FP.

Os resultados foram analisados através de regressão linear, coeficiente de correlação de Pearson, teste do qui-quadrado e análise de variância de um via (one-way ANOVA), seguido do teste de Tukey. Os resultados foram significativos quando $P<0,05$. Foi utilizado o programa PRISM $^{\circledR}$, versão 3.02, 1999, da GraphPad Software Incorporated.

\section{RESULTADOS}

O FP dos 64 pacientes avaliados apresentou média de 1.194,38 mL/ $\min \pm 700,57 \mathrm{~mL} / \mathrm{min}$, tendo como valor mínimo 317,25 e máximo de $3.181,36 \mathrm{~mL} / \mathrm{min}$. A mediana foi de $1.093,52 \mathrm{~mL} / \mathrm{min}$, o $25^{\circ}$ percentil foi de $672,79 \mathrm{~mL} / \mathrm{min}$ e o $75^{\circ}$ percentil foi de $1.523,25 \mathrm{~mL} / \mathrm{min}$.

Quando correlacionadas as provas de perfil laboratorial hepático e o FP, somente a GGT apresentou correlação positiva significativa $(P=0,0379 ; \mathrm{r}=+0,3214)$, conforme observado na Figura 2 . No cotejo dos quartis, também somente a GGT mostrou resultado significativo, donde se constatou que o quarto quartil (4Q) foi diferente estatisticamente do primeiro quartil (1Q) e do terceiro quartil (3Q), conforme ilustrado na Figura 3.

\section{DISCUSSÃO}

Atualmente, muito se tem estudado sobre o FP nos pacientes com hipertensão portal ${ }^{(11,18,19,20,21,28)}$. Há referências de que a esquistossomose hepatoesplênica é a principal causa de hipertensão portal no mundo ${ }^{(24,30)}$. Nota-se, contudo, que os estudos na literatura correlacionando o FP com a função hepática abordam destacadamente o fígado cirrótico $^{(11,18,20,21,28)}$. Provavelmente, isso decorre do fato de ser a cirrose mais comum nos países desenvolvidos e que nitidamente são pacientes com comprometimento hepático mais acentuado.

Já foi demonstrado que os pacientes esquistossomóticos apresentam FP superior aos indivíduos normais (controle) ${ }^{(4,5,19)}$. Desta forma, teriam provavelmente condição de manter boa perfusão hepática por vencer o bloqueio pré-sinusoidal e preservar a função do fígado ${ }^{(19)}$.

No presente trabalho, somente a GGT apresentou correlação positiva significativa com o FP. Da mesma forma, no cotejo dos quartis, o nível sérico da GGT no 4Q foi significativamente maior em comparação com o 1Q e o 3Q. Tal elevação da atividade sérica da GGT nos pacientes com esquistossomose hepatoesplênica já havia sido relatada na literatura. MANSOUR et al. ${ }^{(22)}$ sugeriram que a avaliação da GGT associada à da monoamina-oxidase (MAO) aperfeiçoaria a capacidade de diferenciar bioquimicamente entre a

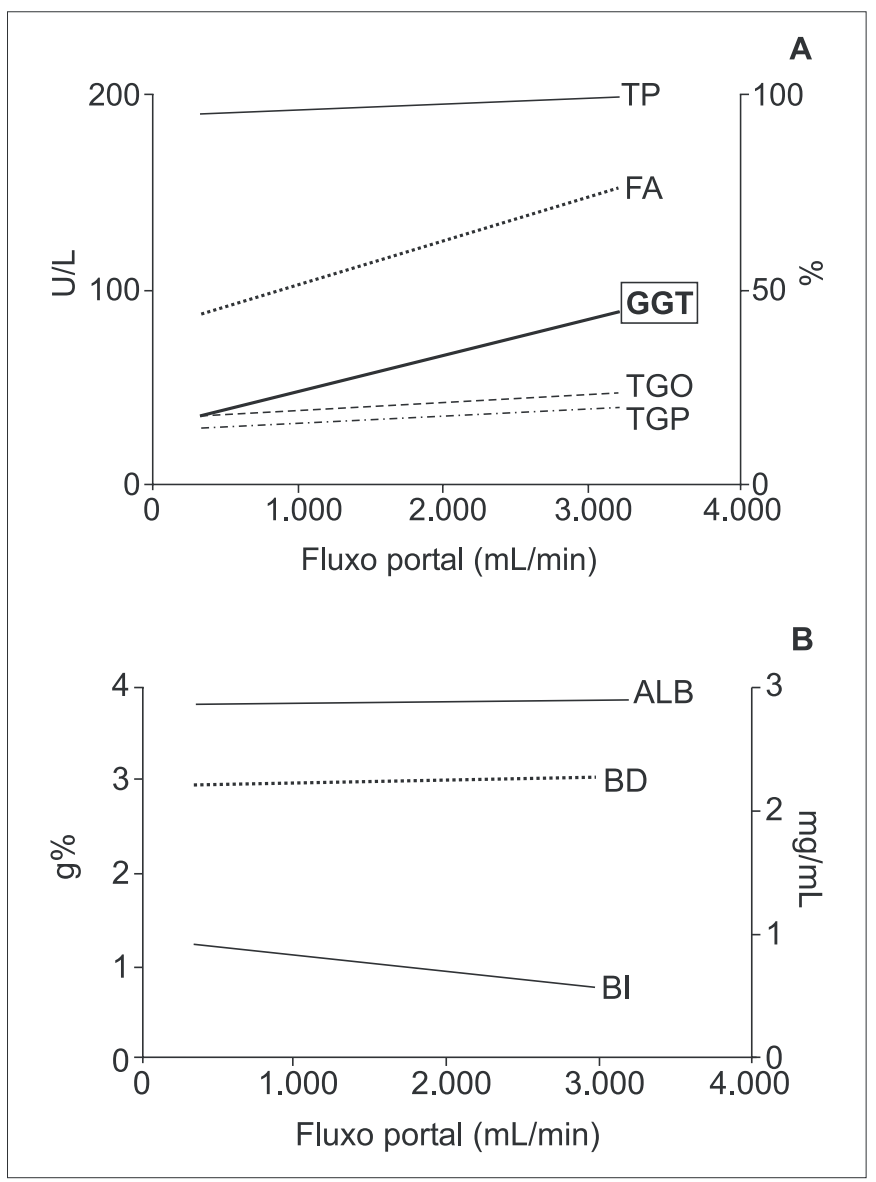

FIGURA 2 - Representação gráfica demonstrando a correlação entre o fluxo portal ( $\mathrm{mL} / \mathrm{min})$ e as diferentes variáveis do perfil hepático. A: TGO = transaminase glutâmico-oxalacética $(\mathrm{U} / \mathrm{L})$; TGP = transaminase glutâmico-pirúvica $(\mathrm{U} / \mathrm{L})$; $\mathrm{TP}=$ atividade de protrombina $(\%) ; \mathrm{FA}=$ fosfatase alcalina $(\mathrm{U} / \mathrm{L}) ; \mathrm{GGT}=$ gama-glutamil transferase $(\mathrm{U} / \mathrm{L})$. B: $\mathrm{BD}=$ bilirrubina direta $(\mathrm{mg} / \mathrm{mL}) ; \mathrm{BI}=$ bilirrubina indireta $(\mathrm{mg} / \mathrm{mL}) ; \mathrm{ALB}=$ albumina $(\mathrm{g} \%)$.

infecção precoce pelo Schistosoma mansoni e a forma crônica hepatoesplênica com seu associado envolvimento hepático.

A GGT está presente em quantidades decrescentes no túbulo proximal do néfron, fígado, pâncreas (dúctulos e células acinares) e intestinos. No entanto, a GGT sérica ativa é originária primariamente do fígado ${ }^{(35)}$.

Consideram-se causas mais importantes da elevação da GGT a estimulação crônica do sistema microssomal (ocasionado pela indução e liberação da GGT da fração microssomal do hepatócito) e a presença de colestase.

A causa microssomal já foi afastada em pacientes portadores da forma hepatointestinal da esquistossomose, mas não da forma hepatoesplênica ${ }^{(23)}$. 


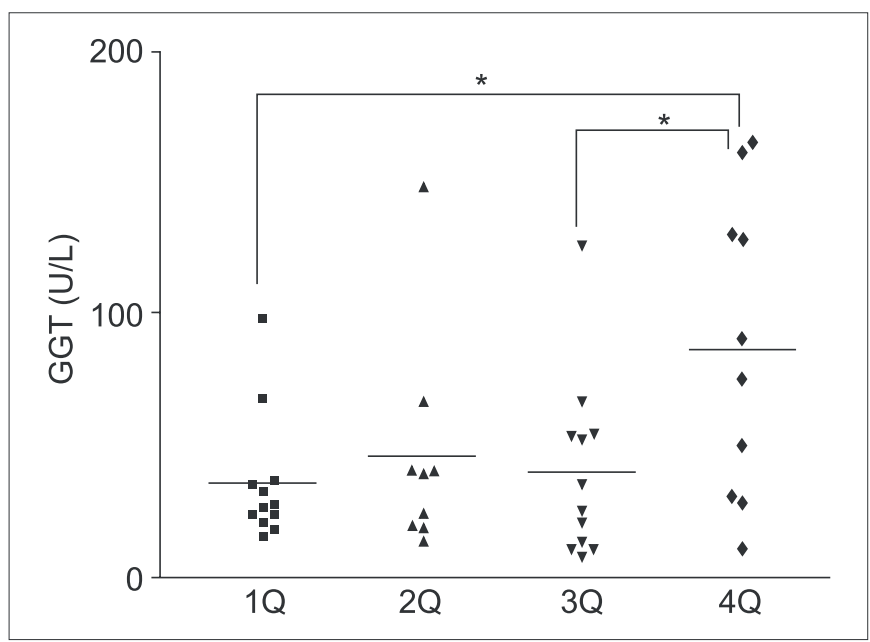

FIGURA 3 - Representação gráfica da comparação dos valores da gama-glutamil transferase (GGT) nos quartis do fluxo sangüíneo portal $(\mathrm{mL} / \mathrm{min})$. As barras horizontais correspondem às médias. $* P<0,05$.

A segunda causa de aumento da GGT é a colestase, melhor avaliada junto com a $\mathrm{FA}^{(27,34)}$. Nesta pesquisa, ficou evidente que a FA acompanha o aumento da GGT em relação ao FP (Figura 2A). No entanto, essa alteração mostrou tendência, não apresentando resultado estatisticamente significativo $(P>0,05)$. Provavelmente, este fato ocorreu porque a GGT foi mais sensível do que a FA em detectar colestase. Alguns estudos que corroboram com tal assertiva demonstraram que a GGT está 12 vezes mais alta na colestase e a FA apenas 3 vezes ${ }^{(3,32)}$. Diversos autores propuseram que as alterações na árvore biliar, decorrentes da fibrose do espaço porta, poderiam ser o substrato anatômico para o aumento de FA e GGT nos pacientes com esquistossomose hepatoesplênica ${ }^{(6,13,16,23)}$

A análise da composição do 4Q, que apresentou os maiores valores de GGT, revela que este é formado em sua maior parte por pacientes não operados, conforme ilustrado na Figura 1 ( $81,25 \%$ - 13 pacientes). Pode-se pensar, desta forma, que a elevação de GGT no 4Q foi devida aos altos valores de GGT dos pacientes com esquistossomose hepatoesplênica não submetidos a cirurgia e, portanto, com maior comprometimento hepático (maior congestão).

Poderia ser questionado se a carga parasitária alteraria os níveis de GGT. No entanto, recentemente, AMARAL et al. ${ }^{(2)}$ demonstraram não haver correlação significativa entre a carga parasitária e os níveis séricos de GGT. A relação encontrada por esses autores entre GGT elevada e índice de protrombina elevado poderia sugerir que a GGT se alteraria em formas mais avançadas da doença e não como resultado de indução microssomal. Além disso, a elevação da GGT, para aqueles pesquisadores, poderia estar presente antes do estabelecimento da forma hepatoesplênica. Os resultados daquele trabalho também evidenciam que a GGT sérica é mais sensível do que a FA em sugerir colestase.

Se a elevação de GGT, encontrada nos resultados do presente estudo, fosse ocasionada pelas alterações na árvore biliar decorrente da fibrose do espaço porta, seria de se esperar que essas também estivessem presentes nos demais quartis. Isso porque, possivelmente, tratam-se de pacientes com esquistossomose hepatoesplênica com semelhantes graus de fibrose no espaço porta. Entretanto, não é o que se nota neste trabalho, no qual os grupos em que há maior participação de pacientes que foram operados apresentam menores valores de GGT do que o 4Q.

WIDMAN et al. ${ }^{(39)}$ demonstraram, por análise ultra-sonográfica, que há redução significativa no calibre da veia porta em pacientes operados por DAPE em relação a pacientes não-operados acometidos da forma hepatoesplênica da esquistossomose. Não obstante, relacionaram essa diminuição do calibre no pós-operatório à diminuição da pressão do FP.

É provável que a fibrose do espaço porta não seja a única responsável pelo grau de colestase encontrado na casuística deste trabalho, e que o regime de maior fluxo e pressão nos ramos da veia porta também concorra para a colestase. Contudo, a hipótese de indução microssomal como causa da elevação da GGT no paciente com esquistossomose hepatoesplênica não foi devidamente afas$\operatorname{tada}^{(23)}$. O que fica evidente, entretanto, é que a elevação da GGT tem correlação positiva com valores do FP.

A análise das outras variantes do perfil hepático não revelou alterações, o que sugere que a função hepatocelular está preservada ou minimamente comprometida na HPE, conforme já cabalmente demonstrado ${ }^{(7)}$. Desta forma, relaciona-se o comprometimento hepático nesta patologia como de ordem predominantemente vascular.

Em conclusão, pode-se inferir que, nesta casuística de pacientes com HPE: 1) quanto maior o FP, maior foi o nível sérico de GGT; 2) a GGT foi a variável da avaliação do perfil hepático mais representativa da influência do FP na atividade funcional hepática nos pacientes com esquistossomose hepatoesplênica, e 3) é possível que as cirurgias, através de suas modificações hemodinâmicas (diminuição da congestão), sejam também benéficas por diminuírem o grau de colestase presente ou em regredirem a indução microssomal. Tal possibilidade, no entanto, requer a realização de novas investigações com diferente metodologia para devida ratificação. 
Alves Jr. A, Fontes DA, Melo VA, Machado MCC, Cruz JF, Santos EAS. Schistosomal portal hypertension: influence of the portal blood flow in serum levels of hepatic enzymes. Arq Gastroenterol 2003;40(4):203-208.

ABSTRACT - Aim - To evaluate relation between the portal blood flow and the laboratory hepatic screening in patients with schistosomal portal hypertension. Patients and Methods - Sixty-four patients with schistosomal portal hypertension had studied, being 19 not operated, 23 submitted to esophagogastric devascularization with splenectomy and 22 submitted to distal splenorenal shunt. Evaluated the laboratory hepatic screening through the dosage of albumin, aspartate aminotransferase, alanine aminotransferase, direct bilirubin and indirect bilirubin, alkaline phosphatase, gamma-glutamil transferase and prothrombin time. The portal flow was evaluated for Doppler. The results have been analyzed through linear regression, Pearson correlation coefficient, chi-square and one-way analysis of variance with Tukey's test. Results - It was proven that only gamma-glutamil transferase had significant correlation with the portal flow. In compare of the quartiles, also only gamma-glutamil transferase showed resulted significant, it was evidenced that the fourth quartile, that is bigger portal flow and formed in its majority for patients not operated, also was bigger average of gamma-glutamil transferase and significantly bigger value than first and the third quartiles. Conclusions -1 . The portal blood flow was bigger in patients that the serum level of GGT was bigger; 2. the gamma-glutamil transferase is the variable of the hepatic screening evaluation more representative of the portal flow influence in hepatic functional activity in patients with hepatosplenic schistosomiasis, and 3. probably, the different surgeries through hemodynamics modifications, are beneficial in to diminish the degree of cholestasis or in decrease the microssomal induction.

HEADINGS - Hypertension, portal. Portal vein. Liver function tests.

\section{REFERÊNCIAS BIBLIOGRÁFICAS}

1. Abrantes WL, Drumond DAF. Anastomose espleno-renal distal em esquistossomótico. Revisão de 200 pacientes operados há 11 e 22 anos. Clin Bras Cir $1995 ; 2: 243-54$

2. Amaral ACC, Aguiar LAK, Souza MRA, Toledo CF, Borges DR. Elevação da $\gamma$-glutamiltransferase sérica na hepatopatia esquistossomótica não se correlaciona com a carga parasitária e precede alterações ultra-sonográficas. Arq Gastroenterol 2002;39:27-31.

3. Anciaux ML, Pelletier AG, Attali P, Meduri B, Liguory C, Etienne JP. Prospective study of clinical and biochemical features of symptomatic choledocholithiasis. Dig Dis Sci 1986;31:449-53.

4. Alves MDNT. Fluxometria da veia porta em pacientes com hipertensão portal esquistossomótica [monografia]. Aracaju: Faculdade de Ciências Médicas da Universidade Federal de Sergipe; 1998.

5. Alves Jr A. Hipertensão portal esquistossomótica. Avaliação do fluxo sangüíneo portal antes e após tratamento cirúrgico [tese]. São Paulo: Faculdade de Medicina da Universidade de São Paulo; 1999.

6. Barreto VST, Domingues ALC. Doença hepática na esquistossomose. In Coelho J, editor. Aparelho digestivo. Clínica e cirurgia. São Paulo: Medsi; 1996. p.1017-84.

7. Capacci MLL. Aminograma plasmático e função hepática na esquistossomose hepatoesplênica [dissertação]. São Paulo: Faculdade de Medicina da Universidade de São Paulo; 1984.

8. Castelo L, Reis M, Aguiar R. Ecografia Doppler a cores. Aplicações clínicas. Acta Med Port 1996;9:177-83

9. Cerri GG. Contribuição da ultra-sonografia no diagnóstico da forma hepatoesplênica da esquistossomose mansônica. [tese]. São Paulo: Faculdade de Medicina da Universidade de São Paulo; 1984

10. Coutinho AD, Domingues ALC. Esquistossomose mansoni. In: Dani R, Castro LP, editores. Gastroenterologia clínica. Rio de Janeiro: Guanabara Koogan; 1993. p.1697-728.

11. De Vries PJ, Hoekstra JBL, De Hooge P, Van Hattum J. Portal venous flow and flow-up in patients with liver disease and healthy subjects. Assessments with duplex Doppler. Scand J Gastroenterol 1994;29:172-7.

12. Friedman SL. Cirrose hepática e suas principais seqüelas. In: Bennett JC, Plum F, editores. Cecil Tratado de medicina interna. Rio de Janeiro: Guanabara Koogan; 1997. p.871-80.

13. Galvão LRR. Alterações radiológicas das vias biliares intra-hepáticas na esquistossomose mansônica. Estudo com a colangiografia endoscópica retrógrada [dissertação]. Recife: Universidade Federal de Pernambuco; 1987.

14. Hermann RE, Henderson JM, Vogt DP, Mayes JT, Geisinger MA, Agnor C. Fifty years of surgery of portal hypertension at the Cleveland Clinic Foundation. Ann Surg 1995;221:459-68.

15. Hill MC, Dach JL, Swaker TH. Ultrasonography in portal hypertension. Clin Gastroenterol 1985; 14:83-104.

16. Hino S, Kakutani H, Ikeda K, Uchiyama Y, Sumiyama K, Kuramochi A, Kitamura Y, Matsuda K, Arakawa H, Kawamura M, Masuda K, Suzuki H. Hemodynamic assessment of the left gastric vein in patients with esophageal varices with color
Doppler EUS: factors affecting development of esophageal varices. Gastrointest Endosc 2002;55:512-7.

17. Kardorff R, Döhring E. Ultrasound diagnosis of bilharziasis. Ultraschall Med 2001;22:107-15.

18. Kawasaki T, Moriyasu F, Kimura T, Someda H, Tamada T, Yamashita Y, Ono S, Kajimura K, Hamato N, Okuma M. Hepatic function and portal hemodynamics in patients with liver cirrhosis. Am J Gastroenterol 1990;85:1160-4.

19. Kazmirik M, De Capua Neto A, Favero SSG, Francisco LDR, Szutan A, De Capua Jr A. Estudo comparativo do fluxo portal em portadores de cirrose e esquistossomose através de ecodoppler. Acta Cir Bras 1994;9:38-43.

20. Lacy AM, Navasa M, Gilabert R, Brú C, García-Pagán JC, García-Valdecasas JC, Grande L, Feu F, Fuster J, Terés J, Visa J, Bosch J. Long-effects of distal splenorenal shunt on hepatic haemodynamics and liver function in patients with cirrhosis: Importance of reversal of blood flow. Hepatology 1992;15:616-22.

21. Ljubicic N, Duvnjak M, Rotkvic I, Kopjar B. Influence of the degree of liver failure on portal blood flow in patients with liver cirrhosis. Scand J Gastroenterol 1990;25:395-400

22. Mansour MM, Farid Z, Bassily S, Salah LH, Watten RH. Serum enzyme tests in hepatosplenic schistosomiasis. Trans R Soc Trop Med Hyg 1982;76:109-11.

23. Martins RD, Borges DR. Ethanol challenge in non-alcoholic patients with schistosomiasis. J Clin Pathol 1993;46:250-3.

24. Mies S, Almeida CG, Raia SMA. Hipertensão portal. In: Netto AC. Clínica cirúrgica. São Paulo: Sarvier; 1994. p.729-62.

25. Miller O, Gonçalves RR, Penteado JF, Porto JAF, Nery ALB, Pecego GF, Ferreira AC, Arantes MR, Alves JMR, Barreto CM, Granato PD, Freier MK. Laboratório para o clínico. Rio de Janeiro: Atheneu; 1977. p.104.

26. Moriyasu F, Ban N, Nishida O, Nakamura T, Miyaki T, Uchino H, Kanematsu Y, Koizumi S. Clinical application of an ultrasonic duplex system in the quantitative measurement of portal blood flow. J Clin Ultrasound 1986;14:579-88.

27. Moss DW. Physicochemical and pathophysiological factors in the release of membrane-bound alkaline phosphatase from cells. Clin Chim Acta 1997;257:133-40.

28. Nishihara K, Sakata K, Yagyu T, Nakashima K, Suzuki T. Relationship between peripheral portal blood flow and function in patients with liver cirrhosis. Scand J Gastroenterol 1994;29:859-61.

29. Parise ER, Ferrari Jr AP, Kondo M. Hipertensão portal, encefalopatia e ascite. In: Prado FC, Ramos J, Do Valle JR, editores. Atualização terapêutica. São Paulo: Artes Médicas; 1995. p.328-34.

30. Pugliese V, Machado MCC, Pinotti HW. Hipertensão portal. In: Pinotti HW, editor Tratado de clínica cirúrgica do aparelho digestivo. São Paulo: Atheneu; 1994. p.830-51.

31. Pugliese V. Desconexão ázigo-portal e esplenectomia associadas à escleroterapia endoscópica no tratamento das varizes do esôfago na esquistossomose hepatoesplênica: avaliação de parâmetros clínicos laboratoriais e hemodinâmicos [tese]. São Paulo: Faculdade de Medicina da Universidade de São Paulo; 1996.

32. Ratanasavanh D, Tazi A, Gaspart E. Hepatic gamma-glutamyl transferase release: effect of bile salt and membrane structure modification. Adv Biochem Pharmacol 1982;3:93-103

33. Sabbá C, Weltin GG, Cicchetti DV, Ferraioli G, Taylor KJW, Nakamura T, Moriyasu F, Groszmann RJ. Observer variability in echo-Doppler measurements 
Alves Jr. A, Fontes DA, Melo VA, Machado MCC, Cruz JF, Santos EAS. Hipertensão portal esquistossomótica: influência do fluxo sangüíneo portal nos níveis séricos das enzimas hepáticas

of portal flow in cirrhotic patients and normal volunteers. Gastroenterology 1990;98:1603-11.

34. Schlaeger R, Haux D, Kattermann R. Studies on the mechanism of the increase in serum alkaline phosphatase activity in cholestasis: significance of the hepatic bile acid concentration for the leakage of alkaline phosphatase from rat liver. Enzyme 1982;28:3-13.

35. Siest G, Henry J, Schiele F, Young DS. Interpretation of clinical laboratory tests: reference values and their biological variation. Foster City, CA: Biomedical Publications; 1985.

36. Souza MR, Toledo CF, Borges DR. Thrombocytemia as a predictor of portal hypertension in schistosomiasis. Dig Dis Sci 2000;45:1964-70.
37. Torquato MM, Marinelli ALC, Castro e Silva Jr O. Aspectos fisiopatológicos da síndrome de hipertensão portal. Rev Med Ribeirão Preto 1993;26:355-8.

38. Vezozzo DCP. Avaliação hepática e hemodinâmica com Doppler duplex na esquistossomose mansônica [tese]. São Paulo: Faculdade de Medicina da Universidade de São Paulo; 1992.

39. Widman A, Oliveira IRS, Speranzini MB, Cerri GG, Saad WA, Gama-Rodrigues J. Hipertensão portal por esquistossomose mansônica hepatoesplênica: efeito da desconexão ázigo-portal com esplenectomia no diâmetro e na velocidade média de fluxo do sistema portal (estudo ultra-sonográfico com Doppler). Arq Gastroenterol 2001;38:19-23.

Recebido em 14/11/2002. Aprovado em 1/8/2003. 